\title{
Hypothalamus-Related Resting Brain Network Underlying Short-Term Acupuncture Treatment in Primary Hypertension
}

\author{
Hongyan Chen, ${ }^{1}$ Jianping Dai, ${ }^{1,2}$ Xiaozhe Zhang, ${ }^{3}$ Kai Wang, ${ }^{1}$ Shuhua Huang, \\ Qingtian Cao, ${ }^{4}$ Hong Wang, ${ }^{5}$ Yuhong Liang, ${ }^{5}$ Chuanying Shi, ${ }^{6}$ Mengyuan Li, ${ }^{1}$ \\ Tingting Ha, ${ }^{1}$ Lin Ai, ${ }^{1,2}$ Shaowu Li, ${ }^{1,2}$ Jun Ma, ${ }^{1}$ Wenjuan Wei, ${ }^{7}$ Youbo You, ${ }^{7}$ Zhenyu Liu, ${ }^{7}$ \\ Jie Tian, ${ }^{7}$ and Lijun Bai ${ }^{8}$ \\ ${ }^{1}$ Department of Radiology, Beijing Tiantan Hospital Affiliated to Capital Medical University, Tiantan Xili No. 6, Beijing 100050, China \\ ${ }^{2}$ Beijing Neurosurgery Institute, Tiantan Xili No. 6, Beijing 100050, China \\ ${ }^{3}$ Department of Pain, Beijing Tiantan Hospital Affiliated to Capital Medical University, Tiantan Xili No. 6, Beijing 100050, China \\ ${ }^{4}$ Department of Medicine, China North Vehicle Research Institute Worker's Hospital, Huaishuling No. 4, Fengtai District, \\ Beijing 100072, China \\ ${ }^{5}$ Tiantan Community Health Service Center, Fenchang Hutong No. 57, Dongcheng District, Beijing 100061, China \\ ${ }^{6}$ Ultrasonic Center, Beijing Tiantan Hospital Affiliated to Capital Medical University, Tiantan Xili No. 6, Beijing 100050, China \\ ${ }^{7}$ Institute of Automation, Chinese Academy of Sciences, Beijing 100190, China \\ ${ }^{8}$ The Key Laboratory of Biomedical Information Engineering of Ministry of Education, Department of Biomedical Engineering, \\ School of Life Science and Technology, Xian Jiaotong University, Xian 710049, China
}

Correspondence should be addressed to Jianping Dai; jpdai2008@yahoo.cn and Lijun Bai; bailj4152615@gmail.com

Received 22 February 2013; Revised 31 March 2013; Accepted 11 April 2013

Academic Editor: Baixiao Zhao

Copyright (C) 2013 Hongyan Chen et al. This is an open access article distributed under the Creative Commons Attribution License, which permits unrestricted use, distribution, and reproduction in any medium, provided the original work is properly cited.

\begin{abstract}
The present study attempted to explore modulated hypothalamus-seeded resting brain network underlying the cardiovascular system in primary hypertensive patients after short-term acupuncture treatment. Thirty right-handed patients (14 male) were divided randomly into acupuncture and control groups. The acupuncture group received a continuous five-day acupuncture treatment and undertook three resting-state fMRI scans and 24-hour ambulatory blood pressure monitoring (ABPM) as well as SF-36 questionnaires before, after, and one month after acupuncture treatment. The control group undertook fMRI scans and 24hour ABPM. For verum acupuncture, average blood pressure (BP) and heart rate (HR) decreased after treatment but showed no statistical differences. There were no significant differences in BP and HR between the acupuncture and control groups. Notably, SF-36 indicated that bodily pain $(P=0.005)$ decreased and vitality $(P=0.036)$ increased after acupuncture compared to the baseline. The hypothalamus-related brain network showed increased functional connectivity with the medulla, brainstem, cerebellum, limbic system, thalamus, and frontal lobes. In conclusion, short-term acupuncture did not decrease BP significantly but appeared to improve body pain and vitality. Acupuncture may regulate the cardiovascular system through a complicated brain network from the cortical level, the hypothalamus, and the brainstem.
\end{abstract}

\section{Introduction}

Hypertension is a common chronic disease affecting onethird of all adults worldwide and causing $51 \%$ of stroke deaths and 45\% of coronary heart disease deaths in 2012 [1]. Investigating hypertension relief has therefore gained increasing attention and interest.
Acupuncture has emerged as a common alternative or complementary therapeutic intervention in western medicine. Reports have stated that acupuncture has certain curative effects for high BP and cardiac pain, with few side effects observed [2]. Despite its public acceptance, unequivocal scientific explanations regarding the mechanism underlying 


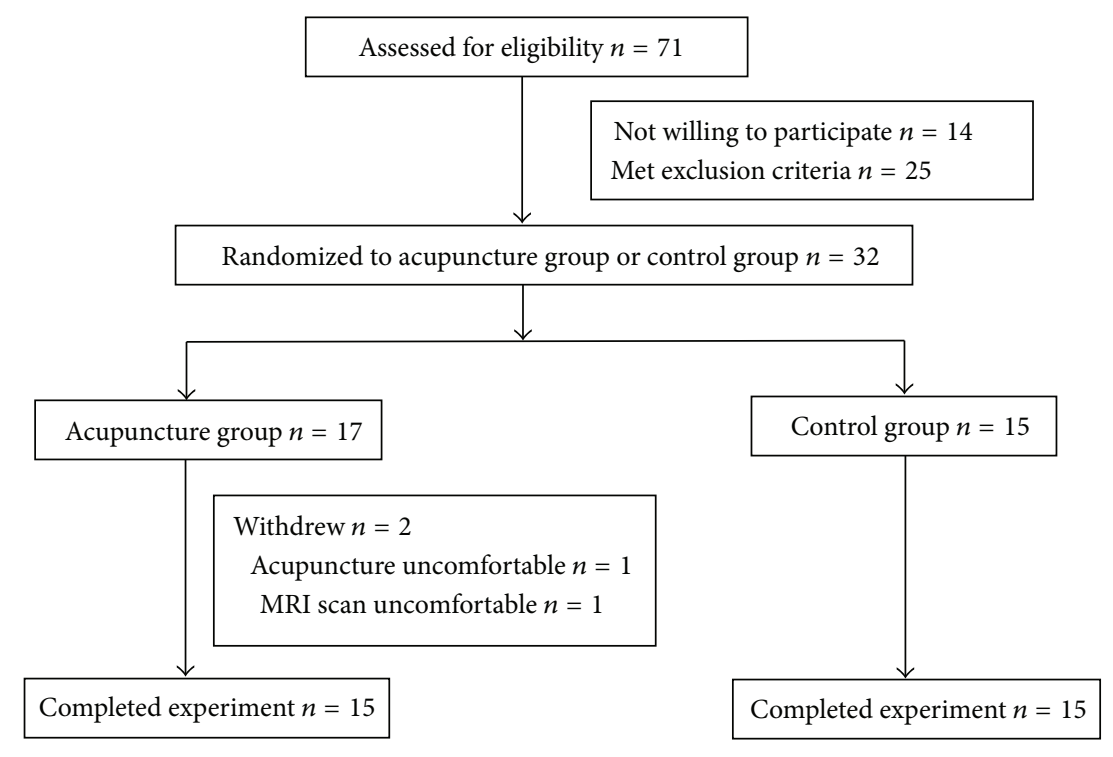

Figure 1: Participants flowchart diagram.

acupuncture in high BP treatment has not been attained and awaits further investigation.

In the recent years, the therapeutic effect of acupuncture in lowering BP has been investigated and discussed in many case reports and small-scale clinical trials [3-7], as well as several large-scale clinical trials on hypertension with acupuncture treatments [8-10]. However, these studies have only focused on the efficacy of acupuncture, and the mechanism of acupuncture in hypertension relief remains unknown.

In the past two decades, converging evidence from fMRI studies has demonstrated that acupuncture stimulation can modulate neural activities in a wide cortical-subcortical network, particularly the limbic system [11-19]. In practice, the well-identified physical effects of acupuncture and its purported clinical efficacy also suggest that it helps maintain a homeostatic balance of the internal state within and across multiple brain systems. Evidence from animal studies has demonstrated that acupuncture stimulation can facilitate the release of certain neuropeptides in the central nervous system (CNS), eliciting profound physiological effects and even activating self-healing mechanisms $[20,21]$. Electroacupuncture studies in rats revealed that both low-frequency and highfrequency stimulation induced analgesia, but differential effects existed in low- and high-frequency acupuncture on the types of endorphins released [20]. Peripheral acupuncture stimulation in deeper areas also activated various brain structures, such as the limbic, hypothalamic, and brainstem neural nuclei [21].

The present study attempted to investigate short-term acupuncture treatment in hypertension relief using resting state fMRI combined with a 24-hour ABPM. The aim was to (i) observe if short-term acupuncture decreased BP of hypertensive patients and improved quality of life and (ii) determine the possible mechanism of short-term acupuncture for treating hypertension in the hypothalamusrelated brain network.

\section{Materials and Methods}

2.1. Participants. We recruited hypertensive participants from the Beijing Dongcheng District Tiantan community health service center and Fengtai District 201 community hospital. The eligibility criteria included (1) clinical diagnosis in line with WHO diagnostic criteria of essential hypertension: systolic blood pressure (SBP) $\geq 140 \mathrm{mmHg}$ and diastolic blood pressure (DBP) $\geq 90 \mathrm{mmHg}$ without antihypertensive drugs, two or more repeated measurements, normal BP now with the use of antihypertensive drugs but with a clear history of hypertension, and species and dose of antihypertensive medicine not changed or suspended or not taking antihypertensive drugs during the study period $[8,9]$; (2) 30-75 years old; (3) without cerebral infarction history and without cerebral infarction by MRI preliminary screening; (4) without nervous system disease, mental disease, diabetes, or other serious illness; (5) no drug addiction history; (6) no MRI contraindication and acupuncture contraindication; (7) no acupuncture experience over the past year; (8) right-handed by Edinburgh handedness questionnaire [19]; (9) provision of informed consent. The exclusion criteria included (1) could not receive acupuncture; (2) any unsuitable situation in the study. The drop-off criteria are as follows: (1) hypertensive crisis or other emergency; (2) could not adhere to acupuncture treatment. The present study was according to the principles of the Declaration of Helsinki (Version Edinburgh 2000).

Of the 71 participants recruited, only 30 (14 male, 3574 years, mean age of $56.73 \pm 9.29$ years) met the eligibility criteria and finished the experiment (Figure 1). All subjects 


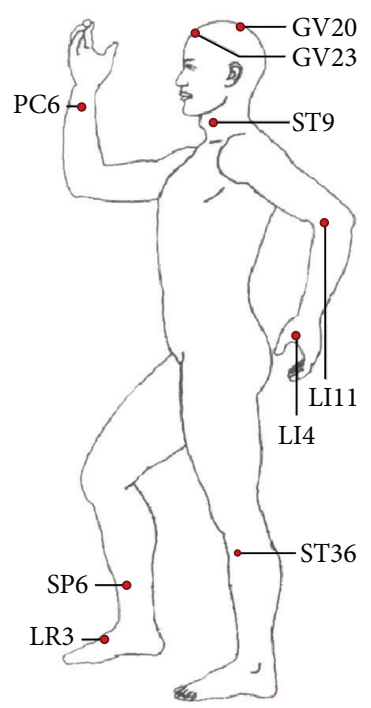

FIGURE 2

were randomly divided into the acupuncture group and the control group, with 15 participants in each.

2.2. Acupuncture Interventions. Participants underwent five acupuncture treatments over five consecutive days, with each session lasting about 30 minutes. During each treatment, seven acupoints used to treat hypertension in clinical settings were selected and needled bilaterally (except single points like GV20 (baihui), GV23 (shangxing), and four points EXHN1 (sishencong)), including ST9 (renying), LI11 (quchi), PC6 (neiguan), LI4 (hegu), ST36 (zusanli), SP6 (sanyinjiao), and LR3 (taichong) [10, 22] (Figures 2 and 3). After local skin disinfection, sterile acupuncture needles $(0.2 \mathrm{~mm}$ in diameter, $40 \mathrm{~mm}$ long, Hua tuo acupuncture needles, Suzhou, China) were inserted into the skin to a depth of $15-50 \mathrm{~mm}$ according to different acupoints and were gently twisted in a mild reinforcing-reducing method 4-6 times till a deqi response was obtained. Control group participants did not receive acupuncture treatment and took medicine according to their original treatment programs. The acupuncture procedure was performed by the same experienced and licensed acupuncturist (15 years of experience) on all subjects.

Figures 2 and 3 show needling acupoints for hypertension treatment. GV20 (baihui) is located in the center of the head; EX-HN1 (sishencong) includes four acupoints located around GV20, each about $3 \mathrm{~cm}$ to GV20; ST9 (renying) is located in the neck, beside the Adam's apple, in the midpoint of the leading edge of the sternocleidomastoid; LI11 (quchi) is located in the outer end of the elbow stripes; LI4 (hegu) is located in the back of the hand, between the first and second metacarpal, radial side of the midpoint of the second metacarpal; PC6 (neiguan) is located in the volar forearm, about $6 \mathrm{~cm}$ above the wrist stripes between tendon and radial measured wrist flexor tendon; ST36 (zusanli) is located in the anterolateral leg, between the tibialis anterior muscle and extensor digitorum longus, about $10 \mathrm{~cm}$ below the knee, about $1.5 \mathrm{~cm}$ from the tibia leading edge; SP6 (sanyinjiao) is located

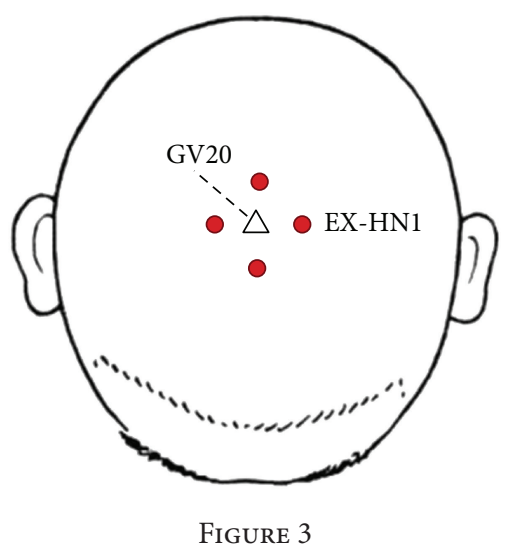

in the medial leg, about $10 \mathrm{~cm}$ above the medial condyle tip; LR3 (taichong) is located in the dorsal foot and the first metatarsal gap rear depression.

2.3. Data Acquisition. The participants were scanned in a 3.0 Tesla Siemens Trio MR whole body scanner. A foam pillow and a band across the forehead were used to fix the head. Resting state functional images were acquired with a singleshot gradient recalled echo planar imaging sequence. The sequence covered the whole brain, axial view, parallel to the AC-PC line, $\mathrm{TR}=2000 \mathrm{~ms}$, $\mathrm{TE}=30 \mathrm{~ms}$, measurement $=$ 240 , resolution $=64 \times 64$, field of view $(\mathrm{FOV})=240 \mathrm{~mm} \times$ $240 \mathrm{~mm}$, flip angle $=90^{\circ}$, slice thickness $=5 \mathrm{~mm}$ without gap, 32 slices, and scan time $=8,06$ minutes. A set of T1weighted high-resolution structural images were collected using a 3D MPRAGE sequence for anatomical localization. $\mathrm{TR}=1900 \mathrm{~ms}, \mathrm{TE}=2.39 \mathrm{~ms}$, field of view $(\mathrm{FOV})=256 \mathrm{~mm} \times$ $256 \mathrm{~mm}$, flip angle $=7^{\circ}$, in-plane resolution $=1 \mathrm{~mm} \times 1 \mathrm{~mm}$, slice thickness: $1 \mathrm{~mm}$ without gap, 32 slices, and scan time = 8,26 minutes.

2.4. Experiment Workflow. The experiment workflow is shown in Figure 3. The first MRI scan adopted the T2WI sequence to exclude participants with cerebral infarction. Participants who met the inclusion criteria underwent resting state fMRI and 3D T1WI structure sequences. The second and third MRI scans were only performed in the resting state $\mathrm{fMRI}$ sequence. The $\mathrm{BP}$ and heart rate $(\mathrm{HR})$ of the participants were measured three times before and after every MRI scan, with the average taken for the three measurements. Participants sat quietly for 5 minutes before each measurement. We used a MOBIL GRAPH sphygmomanometer (Germany) to monitor 24-hour ambulatory blood pressure (ABMP). The BP and HR of the participants were also tested three times before and after every acupuncture treatment, with the average taken for the three recordings.

The acupuncture group received 24-hour ABMP and an MRI scan at multiple time points (before acupuncture, after acupuncture, and one month later). The control group only received 24-hour ABMP and one MRI scan. During the study period, participants maintained their original treatments, and drug type and dosage were not modified except for 


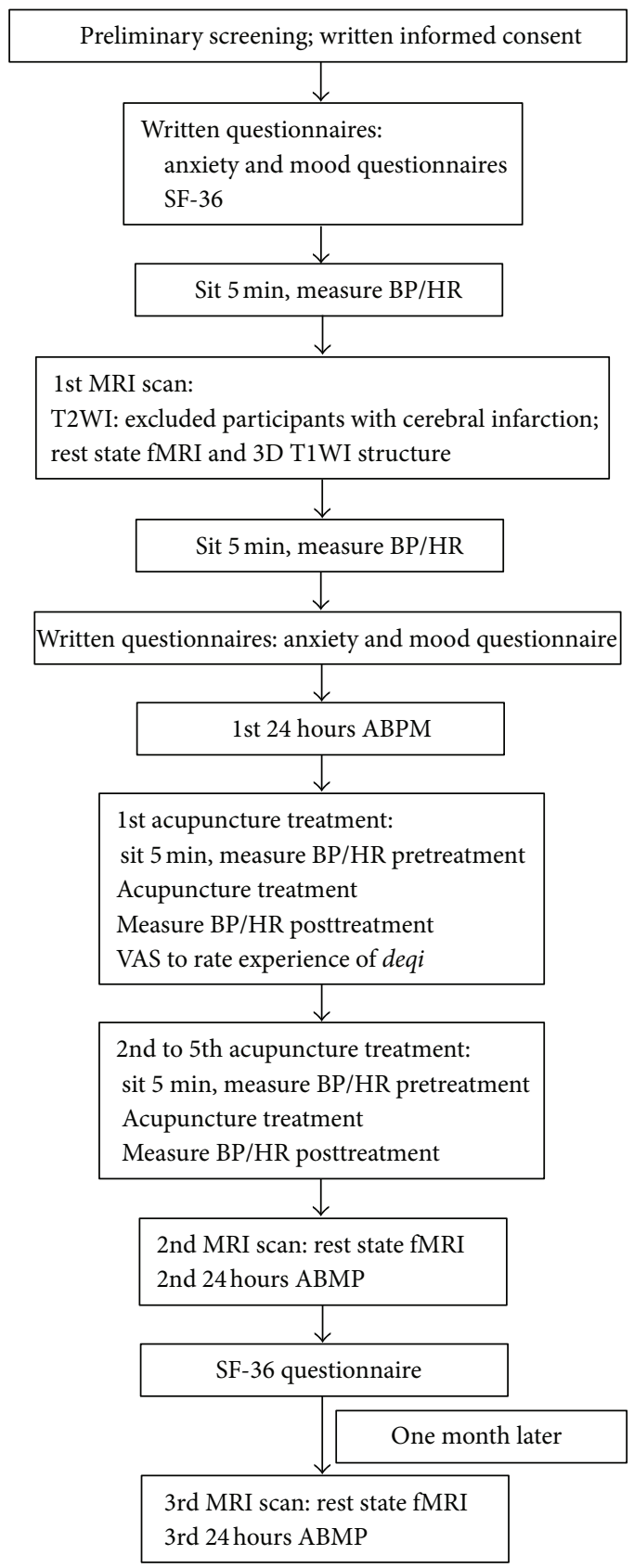

FIGURE 4: Experimental workflow.

emergency situations (SBP $\geq 180 \mathrm{mmHg}, \mathrm{DBP} \geq 110 \mathrm{mmHg}$, or other emergency situations). In case of emergency, the participants were moved into the drop-off group.

At the end of the first acupuncture treatment, the participants completed a questionnaire using a 10-point visual analog scale (VAS) to rate the experience of deqi during intervention. The questionnaire included aching, pressure, heaviness, coolness, soreness, fullness, numbness, warmth, tingling, dull pain, and sharp pain. The VAS was defined as $0=$ no sensation, $1-3=$ mild, $4-6=$ moderate, $7-8=$ strong, $9=$ severe, and $10=$ unbeatable sensation.

Considering that $\mathrm{BP}$ and $\mathrm{HR}$ can be easily affected by psychological factors, such as affective states and anxiety, participants filled out questionnaires for assessments of anxiety (State Trait Anxiety Inventory (STAI)) [23] and affective state (BFS mood survey) [24]. To evaluate quality of life (QoL), subjects also completed questionnaires on MOS item short from health survey (SF-36) [25] before and after acupuncture treatment in the acupuncture group. The control group participants also completed the SF-36 (see Figure 4).

\subsection{Statistical Analysis}

2.5.1. Physiological Data. Since 24-hour ABMP is affected by movement and other factors, the data collected from each participate was not exactly the same. Therefore, we selected independent sample $t$-tests to compare the physiological data and paired $t$-tests to analyze the SF-36 survey. We used SPSS 13.0 for statistical analyses, with $P<0.05$ indicating statistical difference and $P<0.01$ indicating significant statistical difference.

2.5.2. Image Preprocessing. All preprocessing steps were carried out using statistical parametric mapping (SPM5, http://www.fil.ion.ucl.ac.uk/spm/). Functional images were preprocessed using sinc interpolation for slice scan time correction, trilinear sinc interpolation for alignment (motion correction) of functional volumes, and high-pass temporal filtering to $1 \mathrm{~Hz}$ to remove slow drifts in the data. The image data were further processed with spatial normalization based on the MNI space and resampled at $2 \mathrm{~mm} \times 2 \mathrm{~mm} \times$ $2 \mathrm{~mm}$. Poststimuli resting data were also filtered using a band pass filter $(0.01 \sim 0.08 \mathrm{~Hz})$ to reduce low-frequency drift and high-frequency noise. Finally, the functional images were spatially smoothed with a $6 \mathrm{~mm}$ full width at half maximum (FWHM) Gaussian kernel. All resting state functional images were preprocessed using Statistical Parametric Mapping 5 (SPM5) and included motion correction, normalization, and smoothing.

2.5.3. Functional Connectivity Analysis. For each subject, the "seeding" time courses of the hypothalamus were, respectively, cross-correlated with all low-pass filtered voxels to generate functional connectivity maps within each of the three conditions. This approach was termed withincondition interregional covariance analysis (WICA). The resulting correlation coefficient $r$-maps were normalized and corrected to roughly standard normal distributions using methods previously described. The normality of the distribution was then tested using Kurtosis tests $(P<$ $0.001)$. The three $z$-maps of each individual were entered into one-sample $t$-tests, respectively, to determine whether group data was significantly different from zero. For visualization, all connectivity results were transformed into the Talairach stereotactic space and overlaid on MRIcro (http://www.mccauslandcenter.sc.edu/CRNL/) for presentation purposes. All resulting $t$-maps were then cluster-filtered to remove correlations involving less than three contiguous voxels and then superimposed on high-resolution anatomical images using a $P<0.001$ cutoff threshold (uncorrected). 
TABle 1: Physiological data of acupuncture and control groups.

\begin{tabular}{ccccccc}
\hline & $\begin{array}{c}\text { SBP } \\
(\mathrm{mmHg})\end{array}$ & SD & $\begin{array}{c}\text { DBP } \\
(\mathrm{mmHg})\end{array}$ & SD & $\begin{array}{c}\text { HR } \\
(\mathrm{bpm})\end{array}$ \\
\hline ACU & & & & & & \\
B & 127.13 & 5.52 & 81.71 & 8.40 & 71.50 & 8.67 \\
A & 126.74 & 7.93 & 80.86 & 13.36 & 69.83 & 6.53 \\
L & 123.22 & 9.47 & 81.11 & 16.23 & 69.98 & 7.60 \\
CON & 133.40 & 18.58 & 80.93 & 11.49 & 6.43 \\
\hline
\end{tabular}

ACU: acupuncture group; CON: control group; B: before acupuncture; A: after acupuncture; L: one month later.

The above image processing programs were coded in MATLAB7 (MathWorks, Inc.).

\section{Results}

3.1. Physiological Data. Average BP at different time points for the acupuncture and control groups are shown in Table 1. Two independent sample $t$-tests were used to compare the BP and HR between the two groups, $t=-1.529, P=0.127$, with no statistical differences observed in physiological data, indicating comparable balance in the two groups.

Comparison between acupuncture treatment before and after showed that the average SBP, DBP, and HR tended to decrease after acupuncture, although no statistical differences were observed (Table 2). Comparison between the acupuncture and control group showed that average SBP, DBP, and HR demonstrated no statistical differences between the two groups (Table 3 ).

In the SF-36 survey for QoL, participants in the acupuncture group reported increased scores of bodily pain (baseline: mean $\pm \mathrm{SD}, 79.4 \pm 12.3$ versus after acupuncture: mean \pm $\mathrm{SD}, 87.0 \pm 10.6, P=0.005)$ and vitality $(80.0 \pm 15.9$ versus $83.2 \pm 11.9, P=0.036)$. Changes in physical functioning $(92.7 \pm 11.9$ versus $92.7 \pm 11.9)$, role physical $(76.7 \pm 38.3$ versus $78.3 \pm 35.2, P=0.582)$, general health $(67.0 \pm 15.2$ versus $68.7 \pm 13.9, P=0.334)$, social functioning $(96.7 \pm 23.36$ versus $98.3 \pm 19.4, P=0.334)$, role emotional $(91.1 \pm 26.6$ versus $93.3 \pm 25.8, P=0.334)$, and mental health $(70.4 \pm$ 12.9 versus $71.2 \pm 12.5, P=0.082)$ did not differ among participants.

3.2. Connectivity Mapping. At the baseline, the hypothalamus showed prominently spontaneous activations associated with limbic, cortical, and subcortical regions $(P<0.001)$, including the bilateral cerebellum, middle brain, bilateral insula, thalamus, and most of the frontal lobes. These results demonstrated a hypothalamus-anchored resting brain network under baseline conditions. Conversely, spontaneous deactivation was mainly located in the left cerebella vermis, as well as the left superior and right inferior frontal gyrus.

After acupuncture treatment, these spontaneous activation and deactivation networks anchored by the hypothalamus remained relatively stable (Figure 5, $P<0.001$ ). In comparison with baseline conditions, however, we also identified significant changes (both in spatial distributions and response magnitudes) after acupuncture treatment.
There were prominently increased spontaneous activations in the bilateral cerebellum, brainstem, limbic system (bilateral insula, hippocampus, amygdala, and cingulate cortex), bilateral thalamus, and bilateral frontal lobes. Enhanced deactivations were located in the bilateral cerebellum, bilateral frontal lobes, and right parietal gyrus.

Results from after and before acupuncture showed a wide range activation of brain regions (Figure 5). Notably, the most modulated changes were exhibited in the cerebellum, brainstem, insula, and frontal lobe. Increased positive correlations were primarily located in the cerebellum, limbic system (insula, parahippocampal gyrus, and cingulate cortex), bilateral thalamus, and frontal lobes. It is also worth noting that enhanced deactivation was only identified in the temporal lobe, left posterior cingulum, and right parietal lobe. Results from the acupuncture and control groups also showed significant differences in the cerebellum, amygdala, brain stem, and insula, and frontal lobes (see Figure 6).

\section{Discussion}

The present short-term acupuncture intervention study used common acupoints to clinically treat hypertension once a day over five consecutive days. After acupuncture, no statistical differences were found in SBP, DBP, and HR between the before acupuncture and control groups. However, average SBP, DBP, and HR did decrease after acupuncture treatment. The SF-36 survey from the acupuncture group showed statistical differences in body pain and vitality after acupuncture compared to the baseline. Our results did not indicate any differential benefit of short-term acupuncture for controlling $\mathrm{BP}$ and $\mathrm{HR}$, but it may improve quality of life for patients in relation to body pain and vitality. This is the first study to investigate the hypothalamus-anchored brain network involving acupuncture treatments for hypertension.

Several large scale hypertension studies have investigated and discussed the therapeutic effects of acupuncture treatment. Flachskampf et al. [8] randomized 160 hypertensive participants in a single-blind 6-week trial using active or sham acupuncture and found significant $(P<0.001)$ differences in posttreatment BP between the two groups. Yin et al. [9] recruited 41 hypertensive or prehypertensive patients and designed a randomized, double-blind, placebocontrolled trial. All subjects were randomly assigned into real or sham acupuncture groups, and after 8 weeks of intervention, the mean $\mathrm{BP}$ of the acupunctured group was 
TABle 2: Physiological data comparison of acupuncture group (independent $t$-test).

\begin{tabular}{lcccccccccccc}
\hline & \multicolumn{4}{c}{ SBP } & \multicolumn{4}{c}{ DBP } & \multicolumn{4}{c}{ HR } \\
& $t$ value & $P$ value & \multicolumn{2}{c}{$95 \%$ CI } & $t$ value & $P$ value & $95 \%$ CI & $t$ value & $P$ value & $95 \%$ CI \\
\hline B-A & 0.453 & 0.658 & -5.090 & 3.327 & 1.194 & 0.254 & -2.967 & 0.855 & 1.647 & 0.124 & -0.493 & 3.657 \\
B-L & 1.278 & 0.242 & -3.321 & 11.138 & 0.008 & 0.994 & -5.871 & 5.910 & 1.021 & 0.341 & -1.996 & 5.029 \\
A-L & 0.935 & 0.361 & 0.609 & 6.992 & -0.802 & 0.449 & -16.831 & 8.307 & -0.119 & 0.908 & -3.227 & 2.917 \\
\hline
\end{tabular}

B: before acupuncture; A: after acupuncture; L: one month later.

TABLE 3: Physiological data comparisons between acupuncture and control group (independent $t$-test).

\begin{tabular}{|c|c|c|c|c|c|c|c|c|c|c|c|c|}
\hline \multirow[b]{3}{*}{$\mathrm{B}-\mathrm{C}$} & \multicolumn{4}{|c|}{ SBP } & \multicolumn{4}{|c|}{ DBP } & \multicolumn{4}{|c|}{$\mathrm{HR}$} \\
\hline & \multirow{2}{*}{$\begin{array}{l}t \text { value } \\
-1.900\end{array}$} & \multirow{2}{*}{$\begin{array}{c}P \text { value } \\
0.076\end{array}$} & \multicolumn{2}{|c|}{$95 \%$ CI } & \multirow{2}{*}{$\begin{array}{c}t \text { value } \\
0.207\end{array}$} & \multirow{2}{*}{$\frac{P \text { value }}{0.838}$} & \multicolumn{2}{|c|}{$95 \%$ CI } & \multirow{2}{*}{$\begin{array}{l}t \text { value } \\
-0.074\end{array}$} & \multirow{2}{*}{$\frac{P \text { value }}{0.941}$} & \multicolumn{2}{|c|}{$95 \% \mathrm{CI}$} \\
\hline & & & -19.976 & 1.118 & & & -7.031 & 8.602 & & & -6.144 & 5.715 \\
\hline A-C & -1.646 & 0.117 & -19.451 & 2.360 & 0.453 & 0.655 & -6.521 & 10.205 & -0.656 & 0.517 & -7.422 & 3.830 \\
\hline $\mathrm{L}-\mathrm{C}$ & -1.774 & 0.091 & -26.264 & 2.126 & 0.030 & 0.976 & -12.157 & 12.511 & -0.802 & 0.432 & -9.837 & 4.376 \\
\hline
\end{tabular}

B: before acupuncture; A: after acupuncture; L: one month later; C: control group.
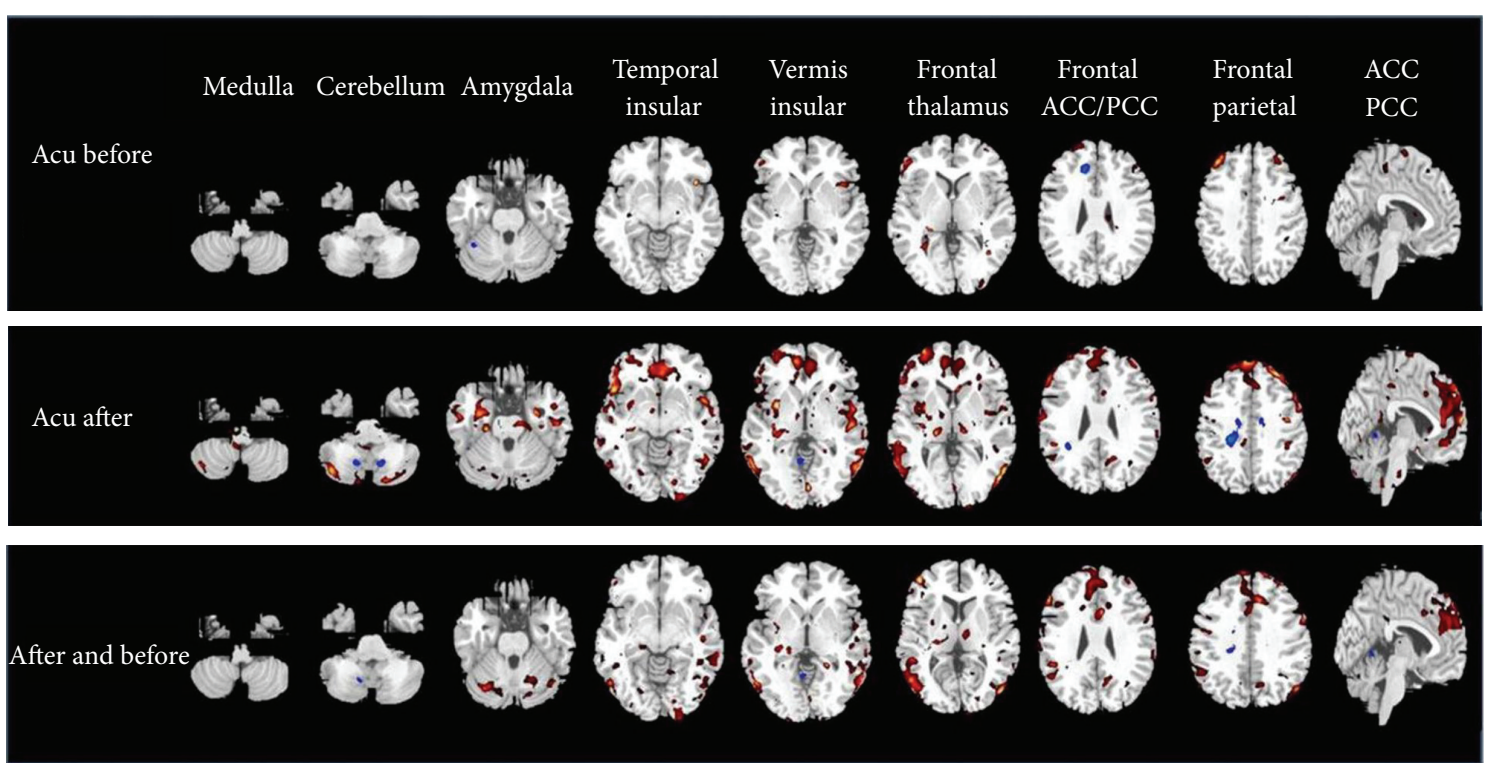

FIGURE 5: Functional connectivity anchored with the hypothalamus after and before acupuncture treatment.
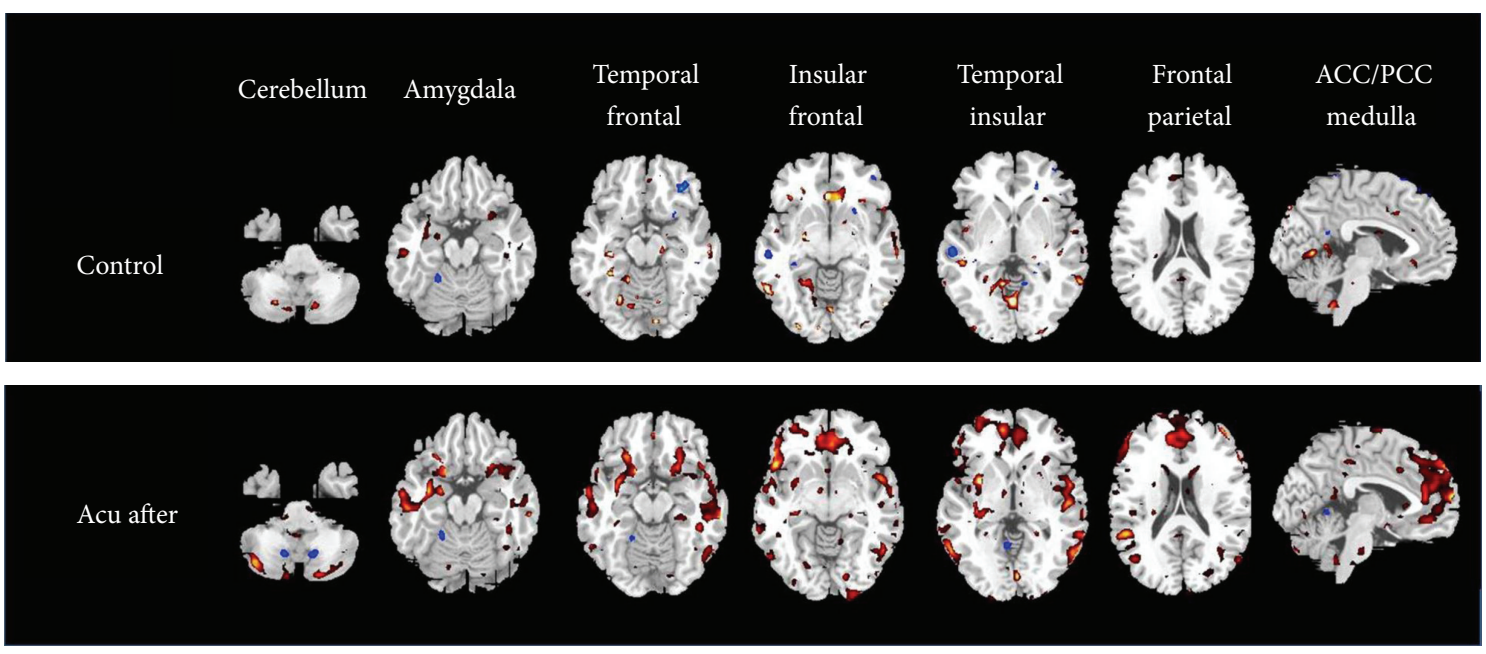

FIGURE 6: Functional connectivity anchored with the hypothalamus resulting from acupuncture control. 
significantly decreased $(P<0.01)$. The Stop Hypertension with Acupuncture Research Program (SHARP) [10] showed there were no significant differences in BP decrease in the individualized traditional Chinese acupuncture group (IND), standardized acupuncture group (STD), and invasive sham acupuncture group (CNTL). Despite there being no statistically significant differences, mean decreases in BP after 10 weeks of treatment were observed between active (IND and STD) and sham acupuncture in comparison to the baseline in the SHARP study. The trials of Flachskampf et al. [8] and Yin et al. [9] showed a decrease in BP after acupuncture, but SHARP [10] only showed a downtrend without statistical differences. In previous studies, we found that the Flachskampf's and Yin's studies were long term with intensive frequency of acupuncture treatment, while the SHARP study was long term with infrequent acupuncture treatment. Acupuncture in our study was short term and intensive, which resulted in no significant differences between acupuncture and control group. Considering that acupuncture has sustained and cumulative effects, short term use may lead to inconsistent results. We therefore speculated that the efficacy of frequent, long-term acupuncture would be better than long interval use or short-term treatment in hypertensive patients.

The cardiovascular center is mainly located in the medulla oblongata and hypothalamus, and these areas are not in the default brain network [26]. Many electrophysiological studies [27-31] have shown that the hypothalamus may be a key hub in the CNS in relation to cardiovascular regulation. Thus, we selected the hypothalamus as the seed point region to explore modulated brain network changes underlying acupuncture hypertension treatment. Our results suggested that increased functional connectivity existed between the hypothalamus and other regions after acupuncture. The functional connection with the hypothalamus was enhanced in some areas, mainly in the bilateral frontal lobe (vmPFC, dlPFC), limbic system (bilateral insula, amygdala, hippocampus, anterior cingulate cortex, and posterior cingulate cortex), bilateral cerebellum, and medulla oblongata. The central regulatory region of the cardiovascular system is very wide from brainstem to cortex, and includes the medulla oblongata, pons (locus coeruleus), midbrain (periaqueductal gray, substantia nigra), and limbic system (hypothalamus, the amygdaloid, subfornical organ, and insula), which together make up a complete network [27]. The medulla oblongata is the basic center, which includes the rostral ventrolateral medulla (vasoconstriction), ventrolateral part of the medulla oblongata (vasodilation), ambiguous nucleus and dorsal nucleus of the vagus nerve (restrains heart function), and tractus solitarius medulla oblongata (afferent nerve terminal) [28]. Past research has indicated that the medulla oblongata regulates the cardiovascular system through neurotransmitters such as glutamate, P substance and $\gamma$-aminobutyric acid [29-31].

Previous studies on electrophysiology have shown that the periaqueductal gray (PAG), hypothalamus, and other parts of the limbic system were important parts of the cardiovascular center [32-40] through different neurotransmitters, adjusting the sympathovagal balance to regulate the cardiovascular system. Many projection fibers are found between the ventrolateral medulla, PAG, and hypothalamus arcuate nucleus for their closely interconnected, and adjusted the cardiovascular system in common [32-34]. The limbic system also plays an important part in BP regulation in the CNS. Some parts are involved in step-up regulation, such as the paraventricular nucleus, lateral hypothalamus/perifornical area, dorsomedial nucleus, ventromedial nucleus, posterior hypothalamic nucleus, lateral septum ventrolateral part/habenular body, subfornical organ, and the central amygdala and insula, while the anteroventral third ventricle area (AV3V area) and the arcuate nucleus participate in decompression regulator, with interactions occurring between these regions $[28,29,35-40]$. Although the role of the cerebellum in cardiovascular regulation is unclear, animal experiments suggest that the neuronal excitability of the fastigial nucleus has the effect of decreasing BP [41-43]. Electrical stimulation of the fastigial nucleus can increase arterial BP and HR, which relieve neuron injury through chronic cerebral ischemia, and was helpful for damaged arterial baroreflex function recovery [41-43]. Normal adults can also present transient changes in physiological indicators after fastigial nucleus electrical stimulation, which suggests a decrease in peripheral vascular flow and an increase in peripheral resistance and a slight increase in heart rate [44]. In addition, the vermiform process of the cerebellum may be important in cerebellum cycle control [45]. Idiaquez et al. [46] reported on vermis and right cerebellar hemisphere resection from cerebellar hemorrhage, where the patient appeared transient orthostatic hypotensive, suggesting that the cerebellum was involved in cardiovascular regulation. We found a negative correlation to the hypothalamus in the fastigial nucleus, which may play a part in the therapeutic action of acupuncture.

Beissner et al. [47] combined cardiac-gated brainstemsensitive fMRI to investigate the mechanism of acupuncture. They found that, from the cortical level, the fMRI group showed significant increases activation in dorsolateral prefrontal gyrus (dlPFC), anterior mid cingulated cortex (ACC), insula, and frontal lobe cortex. Deactivations were found in the ventromedial prefrontal and orbitofrontal cortices (vmPFC/pgACC). They supposed that these areas were involved in acupuncture-induced heart rate changes. Further, as these areas are typical cortical and brainstem centers of pain and autonomic processing, they hypothesized that acupuncture may be a low-intensity deep pain stimulus that can activate autonomic concomitants and exert nonanalgesic effects with therapeutic potential. Our results also indicated a positive correlation with the hypothalamus in vmPFC, ACC, insula, and dlPFC, and these areas may participate in cardiovascular regulation in acupuncture treatment of hypertension and relieve patient pain.

The present study was limited by the small sample size. During our research, patients were found to have different reactions to acupuncture, with some being sensitive to acupuncture and some not. A larger sample size would be necessary to draw more definitive conclusions. Secondly, clinical experience and comparison with large trials indicated that long-term, frequent acupuncture may have good efficacy. The present study indicated that short-term acupuncture 
did not improve the BP of primary hypertensive patients. Finally, nonbrainstem sensitive fMRI sequences were used here. Because the main cardiovascular center is located in the brainstem, a more brainstem sensitive sequence is needed to investigate the mechanism of acupuncture for treating hypertension.

\section{Conclusions}

According to our results, short-term acupuncture did not decrease BP significantly but may have improved body pain and vitality. Acupuncture may also regulate the cardiovascular system through a complicated brain network from the cortical level, the hypothalamus, and the brainstem. However, the mechanism remains unclear and needs further investigation.

\section{Conflict of Interests}

The authors declare that they do not have conflict of interests.

\section{Acknowledgments}

This study was supported by the National Natural Science Foundation of China under Grant nos. 81071137, 81071217, 61171002, 81071139, and 30770617, the Beijing Nova Program (Grant no. Z111101054511116), Beijing Natural Science Foundation (Grant no. 4122082), open program of the CAS Institute of Automation, and the Technology Development Program of Beijing Municipal Commission of Education (Grant no. KZ201010025025). The authors thank Hui Hu, Ling Pan, and Peng Chen for developing the acupuncture program, as well as Wei You and Jing Wang for acupuncture manipulations. The authors also thank Xiaojuan $\mathrm{Ru}$ and Dongling Sun for their statistical assistance.

\section{References}

[1] World Health Organization, World Health Statistics 2012, World Health Organization, 2012.

[2] "NIH Consensus Conference, Acupuncture," Journal of the American Medical Association, vol. 280, pp. 1518-1529, 1998.

[3] H. Huang and S. Liang, "Acupuncture at otoacupoint heart for treatment of vascular hypertension," Journal of Traditional Chinese Medicine, vol. 12, no. 2, pp. 133-136, 1992.

[4] Y. J. Chiu, A. Chi, and I. A. Reid, "Cardiovascular and endocrine effects of acupuncture in hypertensive patients," Clinical and Experimental Hypertension, vol. 19, no. 7, pp. 1047-1063, 1997.

[5] K. C. Tam and H. H. Yiu, "The effect of acupuncture on essential hypertension," American Journal of Chinese Medicine, vol. 3, no. 4, pp. 369-375, 1975.

[6] W. Guo and G. Ni, "The effects of acupuncture on blood pressure in different patients," Journal of Traditional Chinese Medicine, vol. 23, no. 1, pp. 49-50, 2003.

[7] J. D. Pandian, G. Toor, R. Arora et al., "Complementary and alternative medicine treatments among stroke patients in India," Topics in Stroke Rehabilitation, vol. 19, no. 5, pp. 384-394, 2012.
[8] F. A. Flachskampf, J. Gallasch, O. Gefeller et al., "Randomized trial of acupuncture to lower blood pressure," Circulation, vol. 115 , no. 24, pp. 3121-3129, 2007.

[9] C. Yin, B. Seo, H. J. Park et al., "Acupuncture, a promising adjunctive therapy for essential hypertension: a double-blind, randomized, controlled trial," Neurological Research, vol. 29, supplement 1, pp. S98-S103, 2007.

[10] E. A. Macklin, P. M. Wayne, L. A. Kalish et al., "Stop Hypertension with the Acupuncture Research Program (SHARP): results of a randomized, controlled clinical trial," Hypertension, vol. 48, no. 5, pp. 838-845, 2006.

[11] Z. H. Cho, S. C. Chung, J. P. Jones et al., "New findings of the correlation between acupoints and corresponding brain cortices using functional MRI," Proceedings of the National Academy of Sciences of the United States of America, vol. 95, no. 5, pp. 2670-2673, 1998.

[12] K. K. S. Hui, J. Liu, O. Marina et al., "The integrated response of the human cerebro-cerebellar and limbic systems to acupuncture stimulation at ST 36 as evidenced by fMRI," NeuroImage, vol. 27, no. 3, pp. 479-496, 2005.

[13] W. Qin, J. Tian, L. J. Bai et al., "FMRI connectivity analysis of acupuncture effects on an amygdale-associated brain network," Molecular Pain, vol. 4, article 55, 2008.

[14] L. Bai, H. Yan, N. Li et al., "Neural specificity of acupuncture stimulation at pericardium 6: evidence from an fMRI study," Journal of Magnetic Resonance Imaging, vol. 31, no. 1, pp. 71-77, 2010.

[15] L. Bai, W. Qin, J. Tian et al., "Acupuncture modulates spontaneous activities in the anticorrelated resting brain networks," Brain Research, vol. 1279, no. C, pp. 37-49, 2009.

[16] L. Bai, W. Qin, J. Tian, J. Dai, and W. Yang, "Detection of dynamic brain networks modulated by acupuncture using a graph theory model," Progress in Natural Science, vol. 19, no. 7, pp. 827-835, 2009.

[17] L. Bai, J. Tian, C. Zhong et al., "Acupuncture modulates temporal neural responses in wide brain networks: evidence from fMRI study," Molecular Pain, vol. 6, article 73, 2010.

[18] L. Bai, W. Qin, J. Tian et al., "Time-varied characteristics of acupuncture effects in fMRI studies," Human Brain Mapping, vol. 30, no. 11, pp. 3445-3460, 2009.

[19] Y. B. You, L. J. Bai, R. W. Dai et al., "Acupuncture induces divergent alterations of functional connectivity within conventional frequency bands: evidence from MEG recordings," Plos One, vol. 7, no. 11, Article ID e49250, 2012.

[20] J. S. Han, "Acupuncture: neuropeptide release produced by electrical stimulation of different frequencies," Trends in Neurosciences, vol. 26, no. 1, pp. 17-22, 2003.

[21] G. G. Xing, F. Y. Liu, X. X. Qu et al., "Long-term synaptic plasticity in the spinal dorsal horn and its modulation by electroacupuncture in rats with neuropathic pain," Journal of Pharmacology and Experimental Therapeutics, vol. 321, pp. 1046-1053, 2007.

[22] X. Y. Shen, Meridians and Acupoints, China Press of Traditional Chinese Medicine, Beijing, China, 2nd edition, 2002.

[23] L. Laux, P. Glanzmann, P. Schaffner et al., Das State-TraitAngstinventar, Theotetische Grundlagen und Handanweisung, Beltz, Weinheim, Germany, 1981.

[24] B. A. Abele and W. Brehm, "The conceptualization and measurement of mood: the development of the "Mood Survey"', Diagnostica, vol. 32, pp. 209-228, 1986. 
[25] J. E. Ware and C. D. Sherbourne, "The MOS 36-item shortform health survey (SF-36). I. Conceptual framework and item selection," Medical Care, vol. 30, no. 6, pp. 473-483, 1992.

[26] S. Ji, X. Sun, W. Zhang, Q. Gu, and R. He, "Mechanisms underlying blood pressure control of cardiovascular centers," Journal of Biomedical Engineering, vol. 26, no. 1, pp. 216-220, 2009.

[27] Y. H. Gu, "Mechanisms underlying effects of pressor and depressor areas in limbic forebrain," Journal of Progress in Physiology, vol. 25, no. 4, pp. 205-211, 1994.

[28] Y.Z. Chang and Y. H. Gu, "Functional connections among pressor areas of nucleus hypothalamicus posterior, locus coeruleus and rostral ventrolateral," Journal of Peking University, vol. 24, no. 1, p. 27, 1992.

[29] B. C. Ding and P. Wang, "Role of ventrolateral medullary areas in depressor reflex," Progress in Physiology, vol. 29, no. 3, pp. 271274, 1998.

[30] A. Kantzides, N. C. Owens, R. De Matteo, and E. Badoer, "Right atrial stretch activates neurons in autonomic brain regions that project to the rostral ventrolateral medulla in the rat," Neuroscience, vol. 133, no. 3, pp. 775-786, 2005.

[31] S. N. Yang and S. Wang, "The functional connection of rat habenular nucleus and lateral hypothalamic area in the regulation of cardiovascular activities," Acta Physiologica Sinica, vol. 42, no. 1, pp. 82-88, 1990.

[32] P. Li, S. C. Tjen-A-Looi, and J. C. Longhurst, "Excitatory projections from arcuate nucleus to ventrolateral periaqueductal gray in electroacupuncture inhibition of cardiovascular reflexes," American Journal of Physiology, vol. 290, no. 6, pp. H2535H2542, 2006.

[33] S. C. Tjen-A-Looi, P. Li, and J. C. Longhurst, "Midbrain vlPAG inhibits rVLM cardiovascular sympathoexcitatory responses during electroacupuncture," American Journal of Physiology, vol. 290, no. 6, pp. H2543-H2553, 2006.

[34] P. Li, S. C. Tjen-A-Looi, Z. L. Guo, L. W. Fu, and J. C. Longhurst, "Long-loop pathways in cardiovascular electroacupuncture responses," Journal of Applied Physiology, vol. 106, no. 2, pp. 620-630, 2009.

[35] Y. Wang, Y. Gu, Y. Lu, L. Li, and L. Tan, "Role of substance P in pressor response of lateral hypothamus-perifornical region to glutamate," Chinese Journal of Applied Physiology, vol. 13, no. 4, pp. 337-341, 1997.

[36] X. Ding, J. S. Wu, and Y. H. Gu et at, "Mechanisms underlying pressor response of nucleus dorsomedialis hypothalami to glutamate," Journal of Beijing Medical University, vol. 31, pp. 124126, 1999.

[37] Y. Liang, Y. H. Gu, and Y. C. Lv, "Nucleus paraventricularis and sympathetic nervous system mediate pressor response of nucleus amygdaloideus centralis," Journal of Beijing Medical University, vol. 27, no. 3, pp. 165-167, 1995.

[38] J. S. Wu, Y. H. Gu, L. S. Li et al., "Mechanism underlying pressor response of nucleus ventromedialis is involved in pressor response of central amygdaloid nucleus," Journal of Beijing Medical University, vol. 31, no. 5, pp. 422-425, 1999.

[39] Y.Z. Chang and Y. H. Gu, "Role of brain angiotensin II system in subfornical organ-pressor responses," Acta Physiologica Sinica, vol. 51, no. 1, pp. 38-44, 1999.

[40] W. K. Xu and Y. H. Gu, "Rostral ventrolateral medullasympathetic vasoconstrictor nerve system mediated insular cortex-pressor response," Acta Physiologica Sinica, vol. 46, no. 6, pp. 591-596, 1994.
[41] Z. X. Qian, W. M. Dai, S. G. Fu et al., "Effects of cerebellar fastigial stimulation on the arterial blood pressure and respiratory activities in rabbits," Natural Science Journal of Hainan University, vol. 13, no. 1, pp. 60-64, 1995.

[42] M. Nakai, C. Iadecola, and D. J. Reis, "Global cerebral vasodilation by stimulation of rat fastigial cerebellar nucleus," The American Journal of Physiology, vol. 243, no. 2, pp. H226-235, 1982.

[43] R. F. Zhang, L. R. Gao, N. K. Zhang et al., "Effects of fastigial nucleus electro-stimulation on mortality and arterial baroreflex sensitivity in rats after myocardial infarction," Journal of Shanxi Medical University, vol. 41, no. 7, pp. 588-590, 2010.

[44] J. Wang, X. L. Tian, X. Zhang et al., "Effects of fastigial nucleus stimulation on crucial cardiovascular physiological parameters," Chinese Journal of Applied Physiology, vol. 26, no. 4, pp. 507-509, 2010.

[45] M. J. Holmes, L. A. Cotter, H. E. Arendt, S. P. Cass, and B. J. Yates, "Effects of lesions of the caudal cerebellar vermis on cardiovascular regulation in awake cats," Brain Research, vol. 938, no. 1-2, pp. 62-72, 2002.

[46] J. Idiaquez, R. Fadic R, and C. J. Mathias, "Transient orthostatic hypertension after partial cerebellar resection," Clinical Autonomic Research, vol. 21, no. 1, pp. 57-59, 2011.

[47] F. Beissner, R. Deichmann, C. Henke et al., "Acupuncture-deep pain with an autonomic dimension?" NeuroImage, vol. 60, pp. 653-660, 2012. 


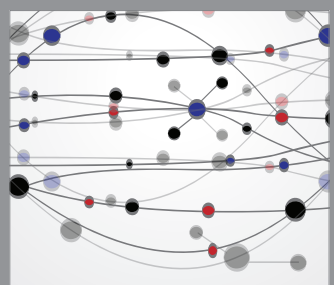

The Scientific World Journal
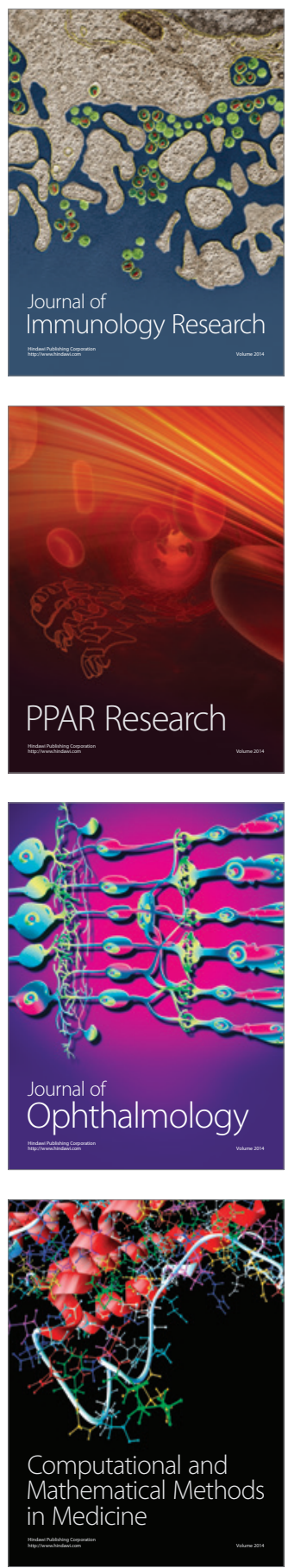

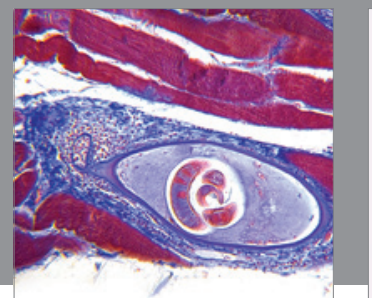

Gastroenterology

Research and Practice
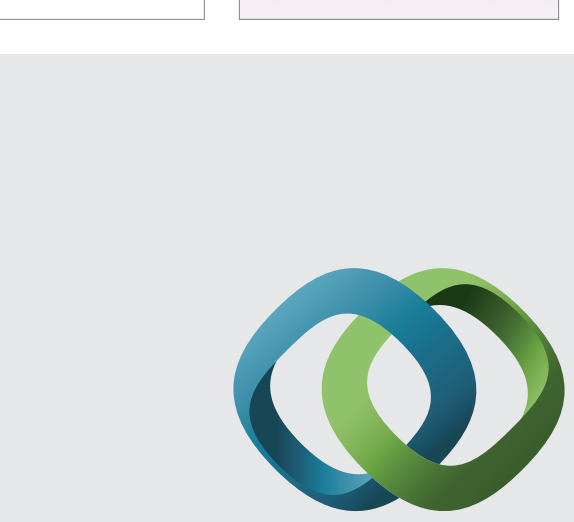

\section{Hindawi}

Submit your manuscripts at

http://www.hindawi.com
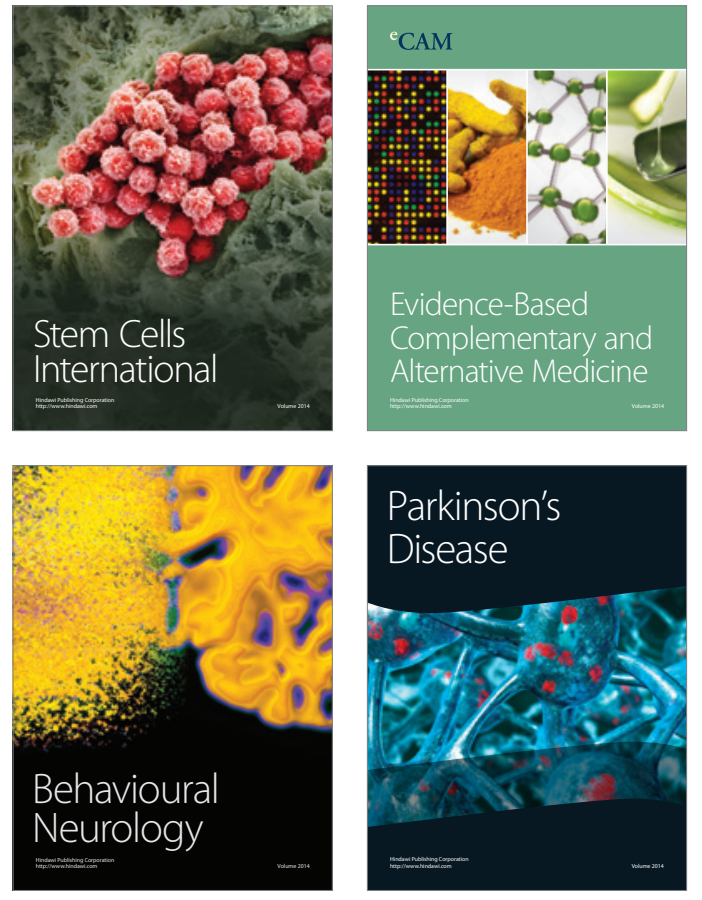
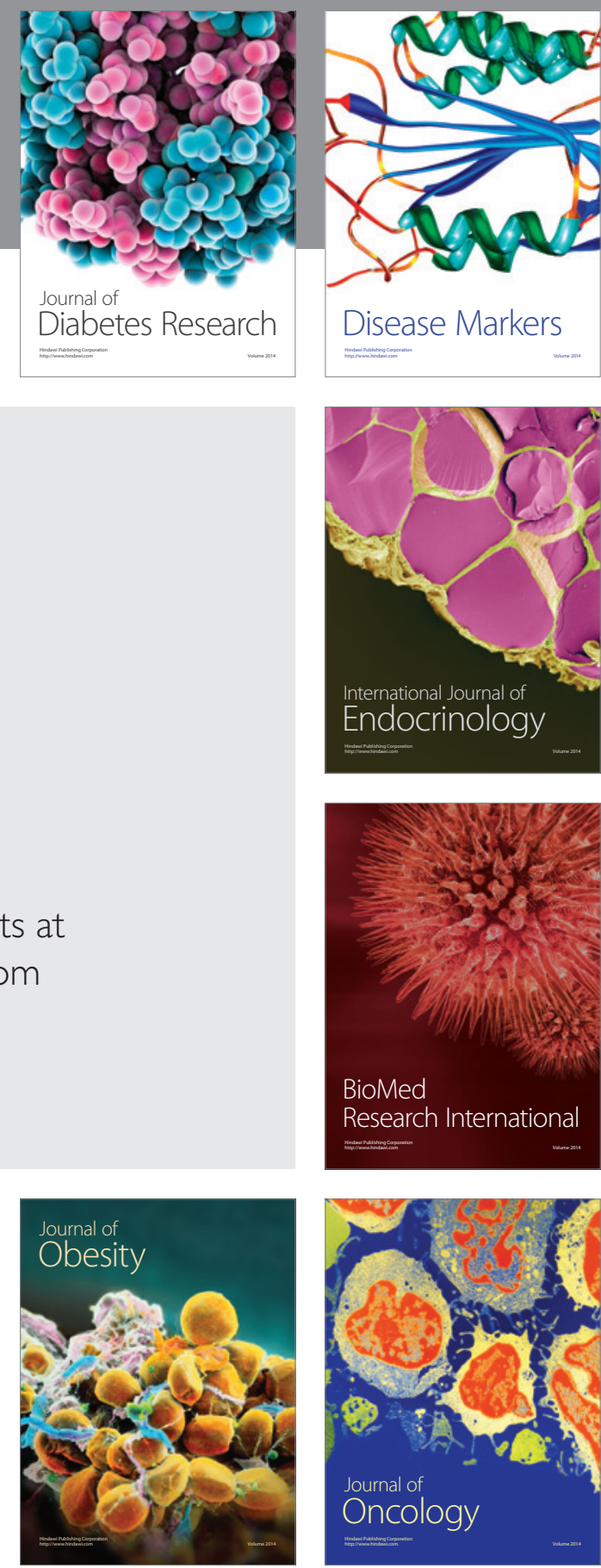

Disease Markers
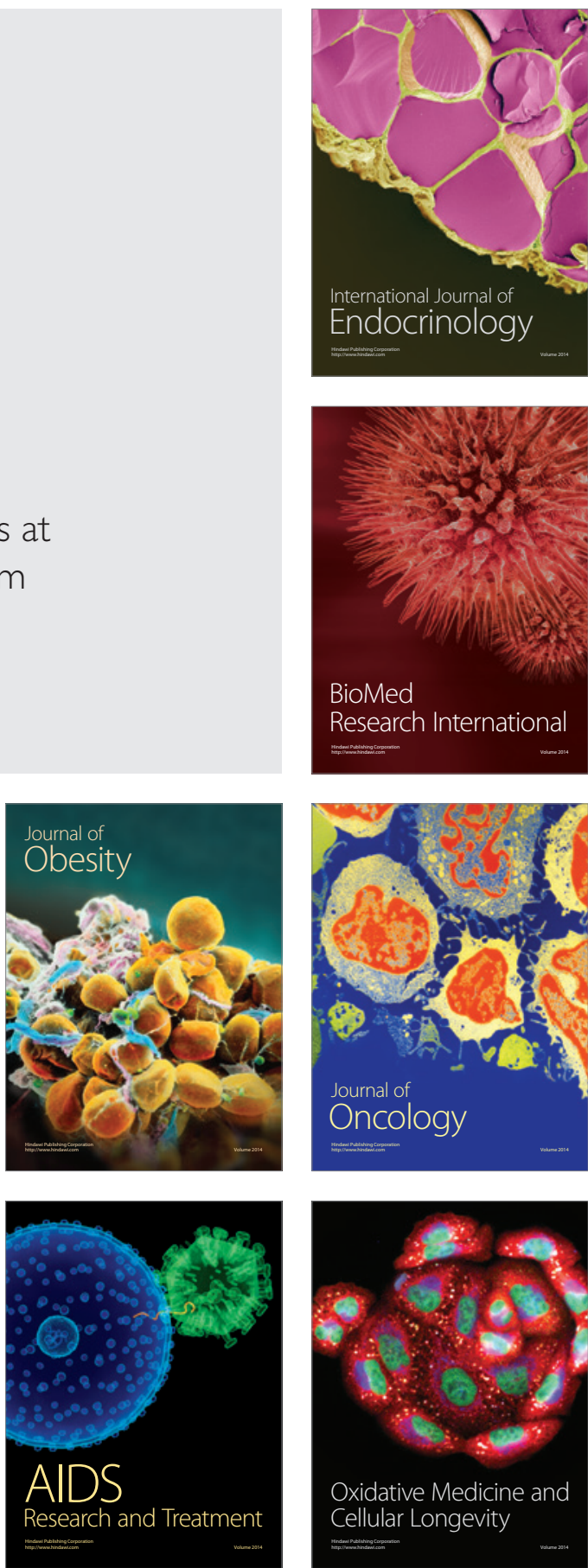